\title{
EXPERIMENTAL STUDIES OF PRE-X VEHICLE HEAT TRANSFER IN PGU-7 TEST FACILITY
}

\author{
R. V. Kovalev, V. V. Kislykh, A. E. Kolozezniy, \\ V. V. Kudryavtsev, V.P. Marinin, V.I. Vlasov, \\ and B. A. Zemliansky
}

TsNIImash, Russia

\begin{abstract}
A number of on-ground tests on aerothermodynamics (ATD) of the Pre-X flight demonstrator at cold supersonic flow regime was made in 2006 in Central Research Institute of Machine Building (TsNIImash, Russia) together with Centre National d'Etudes Spatiale (CNES, France). Objectives of these ATD tests were to obtain experimental data on heat transfer to the Pre-X scaled (1:15) model surface under on-ground experimental conditions to contribute them into the ATD database. The ATD tests included experimental characterization of heat transfer to the model surface using infrared (IR) and calorimetric measurements and were carried out in a piston gasdynamic facility (PGU-7) of TsNIImash. Heat transfer measurements were conducted for a $\mathrm{M}_{\infty}=10$ PGU-7 flow regime at three characteristic Reynolds numbers, three different angles of attack, two sideslip angles, and four flap deflection angles.
\end{abstract}

\section{INTRODUCTION}

The Pre-X demonstrator was the CNES proposal to perform inflight experimentation mainly on reusable thermal protections, ATD, and guidance to secure the second generation of reentry $\mathrm{X}$ vehicles [1]. The general objective of the Pre-X project is to improve European capabilities in the area of atmospheric reentry for space transportation, exploration, and scientific applications. One of general objectives of this project is investigation of different ATD phenomena during atmospheric entry in the hypersonic flow regime $\left(\mathrm{M}_{\infty}>5\right)$.

This paper deals with experimental studies of several ATD phenomena carried out in TsNIImash PGU-7 facility during 2006 test campaign. The test program was intended to experimental characterization of the Pre-X vehicle model surface heat transfer with special attention to windward (wwd) surface heating

This is an Open Access article distributed under the terms of the Creative Commons Attribution-Noncommercial License 3.0, which permits unrestricted use, distribution, and reproduction in any noncommercial medium, provided the original work is properly cited. 
Table 1 Test program

\begin{tabular}{lccrrlcc}
\hline \multicolumn{1}{c}{ Test objectives } & $\alpha$ & $\beta$ & $\delta_{r}$ & $\delta_{l}$ & Re & Transition & $N$ \\
\hline Reynolds number & $45^{\circ}$ & $0^{\circ}$ & $0^{\circ}$ & $0^{\circ}$ & Low/High & Natural & 3 \\
effect + effect & $45^{\circ}$ & $0^{\circ}$ & $5^{\circ}$ & $5^{\circ}$ & Low/Medium & Natural & 2 \\
of flap & $45^{\circ}$ & $0^{\circ}$ & $10^{\circ}$ & $10^{\circ}$ & Low/Medium & Natural & 2 \\
defections & $45^{\circ}$ & $0^{\circ}$ & $15^{\circ}$ & $15^{\circ}$ & Low/Medium/High & Natural & 3 \\
\hline \multirow{3}{*}{ AoA effects } & $40^{\circ} / 50^{\circ}$ & $0^{\circ}$ & $0^{\circ}$ & $0^{\circ}$ & Low & Natural & 4 \\
& $40^{\circ} / 50^{\circ}$ & $0^{\circ}$ & $5^{\circ}$ & $5^{\circ}$ & Medium & Natural & 2 \\
& $40^{\circ} / 50^{\circ}$ & $0^{\circ}$ & $15^{\circ}$ & $15^{\circ}$ & Low/Medium/High & Natural & 6 \\
\hline \multirow{2}{*}{ Sideslip effects } & $45^{\circ}$ & $5^{\circ}$ & $0^{\circ}$ & $0^{\circ}$ & Low & Natural & 1 \\
& $45^{\circ}$ & $5^{\circ}$ & $15^{\circ}$ & $15^{\circ}$ & Low/High & Natural & 3 \\
\hline \multirow{2}{*}{ Aileron effects } & $45^{\circ}$ & $0^{\circ} / 5^{\circ}$ & $10^{\circ}$ & $0^{\circ}$ & Low & Natural & 2 \\
& $45^{\circ}$ & $0^{\circ}$ & $15^{\circ}$ & $5^{\circ}$ & Low/High & Natural & 2 \\
\hline \multirow{4}{*}{ Turbulence effects } & $45^{\circ}$ & $0^{\circ}$ & $0^{\circ}$ & $0^{\circ}$ & High & Forced & 2 \\
& $45^{\circ}$ & $0^{\circ}$ & $5^{\circ}$ & $5^{\circ}$ & Medium & Forced & 1 \\
& $45^{\circ}$ & $0^{\circ}$ & $10^{\circ}$ & $10^{\circ}$ & Medium & Forced & 1 \\
Auxiliary & $0^{\circ}$ & $15^{\circ}$ & $15^{\circ}$ & Medium & Forced & 1 \\
\hline
\end{tabular}

(including lateral side heating); deflected flaps heating; and base heating, first of all, by means of quantitative IR measurements and, to some lesser degree, by using discrete sensors.

Aerothermodynamics phenomena under study were:

- Reynolds number effect on wwd side heating, laminar-turbulent transition (LTT), and viscous interaction on the flaps;

- effect of defection of flaps on their heating and LTT;

- angle of attack (AoA) effects;

- sideslip effects;

- aileron effects (that is the case when lateral force produced by differential deflections of the flaps); and

- turbulence effects due to forced transition induced by a tripping device to characterize an amount of turbulent heating for the facility flow conditions.

The test program included 40 tests at Mach number $\mathrm{M}_{\infty}=10$ for three different Reynolds numbers (Re) as well as for a number of angles of attack and sideslip (Table 1). In Table $1, \alpha$ is the angle of attack; $\beta$ is the sideslip angle; $\delta_{r}$ is the right flap deflection angle; $\delta_{l}$ is the left flap deflection angle; and $N$ is the 
number of tests on given position. Five auxiliary tests were used for checking the reproducibility of test results.

Exact values of the Reynolds numbers were supposed to be chosen during the course of the tests to meet the following demands:

- Low - the test regime at which the flow over all the body surface is laminar (including interaction zone at deflected flaps);

- Medium - the regime with the lowest Reynolds number at which (in case of forced transition) the effective LTT occurs at the model surface (in such a way that turbulent interaction regime takes place at the flaps), and in case of no tripping device, the transient flow regime is realized on the flaps; and

- High - the regime at which the flow before the flaps is transient and the heat fluxes to them achieve maximum level typical for the transient interaction regime.

\section{EXPERIMENTAL TOOLS}

\subsection{PGU-7 Facility}

Experimental specification of the heat transfer characteristics at the Pre-X model surface were conducted in the piston gasdynamic unit - PGU-7 with multicascade compression. Idea of the facility operating is based on well-known method of gas compression by the action of a heavy piston movement in a sequence (cascade) of intervening chambers - multicascade [2]. The facility consists of a driving (compression) section, test section, and exhaust system. Principal chart of PGU-7 compression section is shown in Fig. 1 and includes driven gas balloon (1), barrel (2), heavy piston (3), piston release device (4), pressure chamber (5), valves $(6,7)$, gas accumulation section $(8,9)$, throttle $(11)$, and

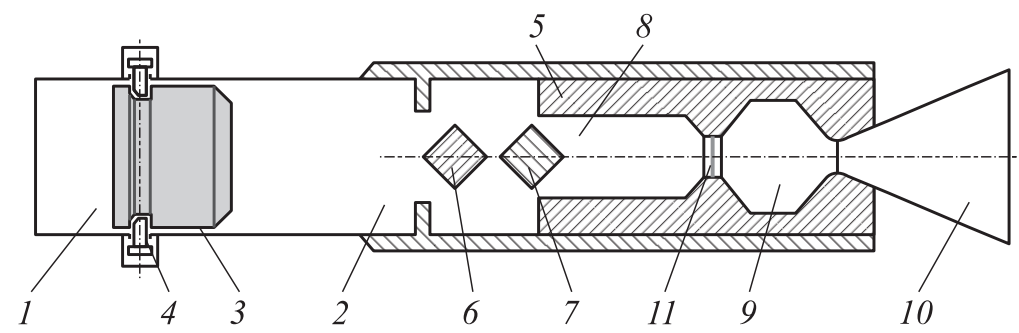

Figure 1 Principal chart of PGU-7 facility driving section 


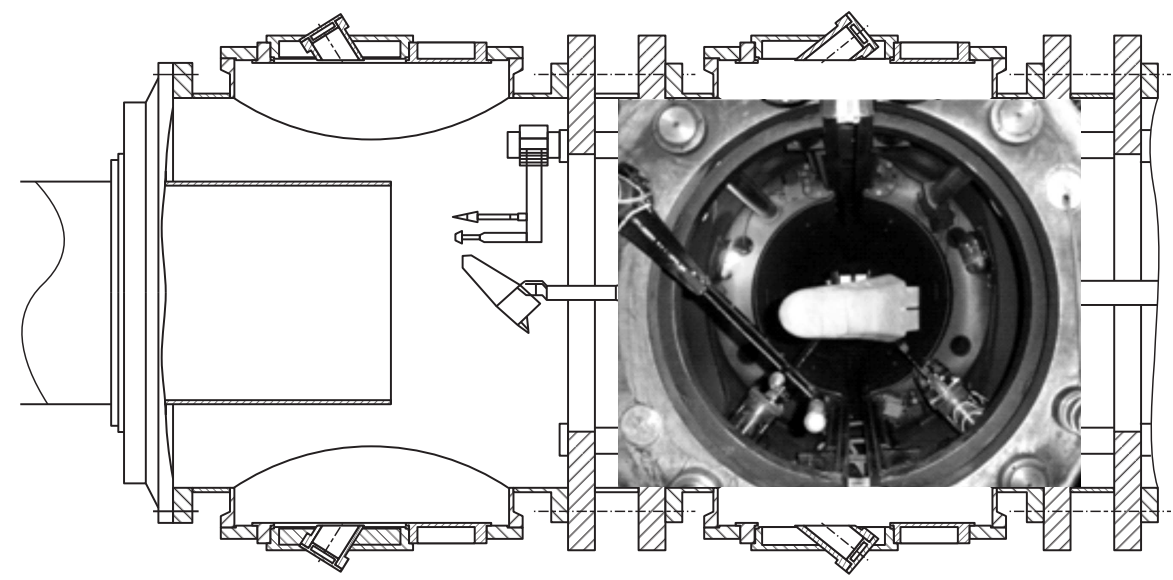

Figure 2 Test section of the PGU-7 facility and nozzle-view to the Pre-X model

nozzle (10). In the present tests, a profiled nozzle with 800-millimeter exit section diameter was used to provide air flow with Mach number $\mathrm{M}_{\infty}=10$. From the nozzle, air flow comes to the test chamber (Fig. 2) where the model is set. Besides, two additional devices were installed apart from the model - pitot and heat flux probes (calorimeter) to register facility flow regime in every test (they also can be seen in the nozzle-view of the model). The model was viewed through the test section windows by IR-cameras that allowed convenient observation of model nose part (upper left window in Fig. 2), wwd surface (lower left window), and base region (lower right window). In the tests with observation of lateral surfaces of the model, it turned to $90^{\circ}$ over horizontal axis.

The PGU-7 facility belongs to a class of impulse facilities with total test duration $\Delta t \leq 0.1-1 \mathrm{~s}$ and the flow parameters, such as reservoir pressure, total temperature, pitot pressure, etc., vary during tests. Temporal behavior of such parameters can be subdivided onto two typical parts: (I) steep growth for about $\sim 5-10 \mathrm{~ms}$, and (II) smooth drop because of gas outflow through the nozzle. Nevertheless, Mach and Reynolds numbers are kept almost constant during useful test time.

Table 2 Flow regimes

\begin{tabular}{lccccc}
\hline \multicolumn{1}{c}{ Test mode } & $\mathrm{M}$ & $\mathrm{Re}_{L}$ & $T_{0}, \mathrm{~K}$ & $P_{\text {pitot }}$, atm & $Q_{w 0}, \mathrm{~kW} / \mathrm{m}^{2}$ \\
\hline Low Re & 10.4 & $4.2 \cdot 10^{5}$ & $1700-700$ & $0.13-0.04$ & $250-35$ \\
Medium Re & 10.4 & $1.1 \cdot 10^{6}$ & $1700-700$ & $0.40-0.11$ & $380-70$ \\
High Re & 10.4 & $1.7 \cdot 10^{6}$ & $1700-800$ & $0.68-0.17$ & $580-100$ \\
\hline
\end{tabular}


Flow regimes used are listed in Table 2 for three selected Reynolds mode. In this table, $\mathrm{M}$ is the flow Mach number; $\mathrm{Re}_{L}$ is the Reynolds number on model length $(L=293 \mathrm{~mm})$; and $T_{0}, P_{\text {pitot }}$, and $Q_{w 0}$ are the total temperature, pitot pressure, and heat flux to stagnation point of copper cold calorimeter $(R$ $=70 \mathrm{~mm}$ ). For three latest values, the ranges of parameter variation during test are presented (maximum value at $t \sim 10 \mathrm{~ms}$ and value at $t=100 \mathrm{~ms}$ ).

\subsection{Test Model}

The model of Pre-X vehicle of scale 1:15 for heat tests was designed and produced in TsNIImash from the AG-4V glass-reinforced resin material with low thermal conductivity and high value of surface emissivity [3] that is required for reliable IR-measurements. An example of graphical representation of $0^{\circ}$-deflected flaps CAD design is presented in Fig. $3 a$. The model made of AG-4V material with fixed lee-side sting is also presented in Fig. $3 b$. Two similar replaceable nose part were manufactured for the tests - one of them was equipped with the low-sized sensors for heat flux measurements and another one was remained smooth (to reduce perturbations of the temperature field introduced by the cold copper calorimeters installed into low-heat conductive hot environment). After manufacturing, the measured maximum surface deviation (body without flaps) from the nominal (CAD) surface was $0.038 \mathrm{~mm}$. The airbrake flaps were also made replaceable (four pairs of flaps with deflection angles of $0^{\circ} / 5^{\circ} / 10^{\circ} / 15^{\circ}$ ) and were produced separately from the model body with further their fitting to the main part of the model. Accuracy of manufacturing of the deflected flaps was checked out after tests with profilometer. Actual values of flap deflection angles are presented in Table 3 ( $\delta$ is the nominal value of flap deflection; and $\delta_{l}$ and $\delta_{r}$ are the actual value of left and right flap deflections in degrees).

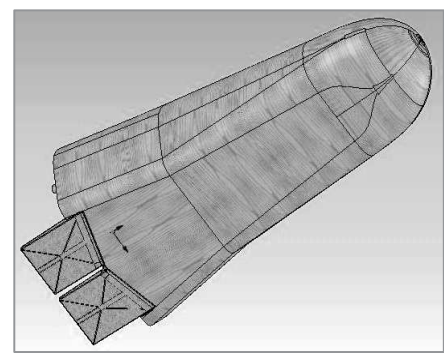

(a)

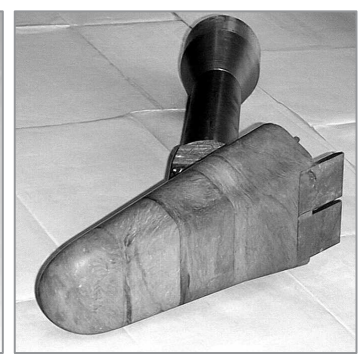

(b)

Figure 3 Model shape derived from the CAD file $(a)$ and model photograph with the sting at its leeside $(b)$ 
Table 3 Actual values of flap deflection angles

\begin{tabular}{rrc}
\hline$\delta$ & $\delta_{l}$ & $\delta_{r}$ \\
\hline $0^{\circ}$ & $-0.60^{\circ} \pm 0.3^{\circ}$ & $0.09^{\circ} \pm 0.3^{\circ}$ \\
$5^{\circ}$ & $4.61^{\circ} \pm 0.3^{\circ}$ & $5.28^{\circ} \pm 0.3^{\circ}$ \\
$10^{\circ}$ & $9.78^{\circ} \pm 0.3^{\circ}$ & $9.91^{\circ} \pm 0.3^{\circ}$ \\
$15^{\circ}$ & $14.9^{\circ} \pm 0.3^{\circ}$ & $14.2^{\circ} \pm 0.3^{\circ}$ \\
\hline
\end{tabular}

In a number of tests (six runs with forced transition on the model surface), the model was equipped with the tripping device - 0.6-millimeter wire twisted around its nose part (at a distance $\sim 70 \mathrm{~mm}$ from the nose) in one layer with the width of twisted strip of $6 \mathrm{~mm}$ (Fig. $4 a$ ). In the base region of the model, the elements reproducing the protuberance elements of the

PRE-X vehicle by sizes were installed (Fig. $4 b$ ): the launcher interface frame structure (1), four RCS (reaction control system) pods (2), the THEFA mirror (3), and two flap central beams (4). These elements were made of AG-4V material and fasten tightly (with pins) at the base surface of the model.

Eight low-sized sensors of the heat fluxes (calorimeters) were installed on the replaceable nose part of the model (TN01-TN08). Position of these sensors is shown in Fig. 5 over IR-data on nose heating projected to the model nose surface as viewed from test facility window in Fig. 2. Besides, in the base region, two thermal resistive sensors (TB1 and TB2) were installed for low temperature measurements.

Deviations in model angle of attack relative to the axis of the PGU-7 test chamber were sufficiently low and were equal to $44^{\circ} 46^{\prime}$ for nominal value of $45^{\circ}$, $39^{\circ} 56^{\prime}$ for AoA of $40^{\circ}$, and $49^{\circ} 36^{\prime}$ for AoA of $50^{\circ}$.

Under aerodynamic forces and moments, the model suffers both static and dynamic responses. Because of firm construction of the sting used in the tests, the static loads bring about quite small displacement of the model, its maximum vertical displacement was about $0.4 \mathrm{~mm}$ and maximum distortion of the attack

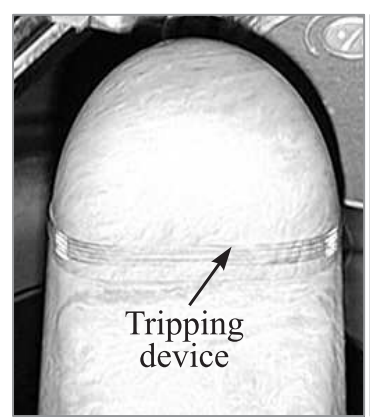

(a)

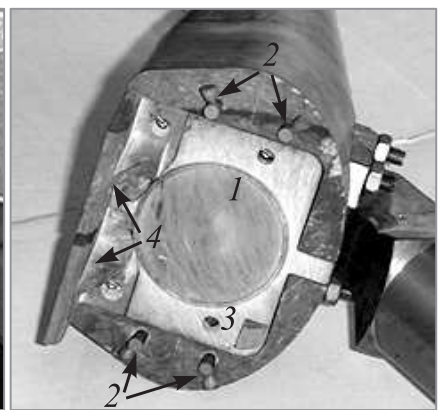

(b)

Figure 4 Tripping device in the nose part $(a)$ and base region close view $(b)$ 


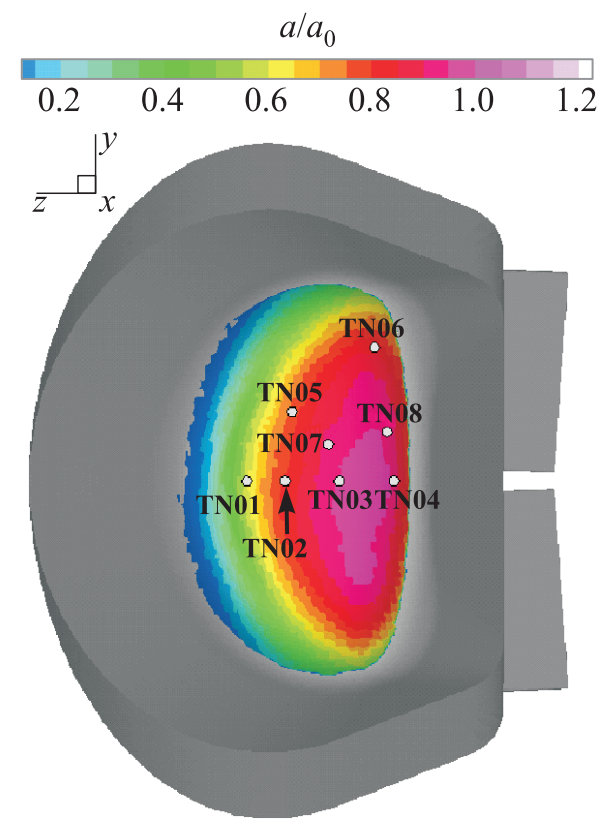

Figure 5 Location on calorimeters at nose part of the model. (Refer R. Kovalev et al., p. 341.)

angle due to aerodynamic moments was $4^{\prime} 36^{\prime \prime}$. Measurements of dynamic response of the model showed that maximum displacement of the junction point under pulse loading is also quite low and is equal to $0.451 \mathrm{~mm}$ and is achieved at $0.0371 \mathrm{~s}$ from the run start.

\section{MEASUREMENT TECHNIQUE}

\subsection{Infrared Measurements}

Two scanning type cameras AGA-780 [4] were used in the tests for IR measurement. These cameras are equipped with replaceable lenses $\left(20^{\circ}\right.$ - and $7^{\circ}$-lenses were used) allowing obtaining different spatial resolution and making fragment shooting of separate parts of the model. Sensitive element of receiving camera takes its maximum sensitivity within the wavelength range $3-5.6 \mu \mathrm{m}$. It is cooled with the liquid nitrogen to exclude background noise. The temperature field is registered in $8192=128 \times 64$ pixels; in that case, single IR-frame is scanned for $40 \mathrm{~ms}$ that is scanning frequency is 25 images/s. AGA-780 temperature sensitivity is $0.1 \mathrm{~K}$ (at temperature level of $30^{\circ} \mathrm{C}$ ). 
During the tests, the model was observed simultaneously from two aspects. The $20^{\circ}$-lens was usually used for observation of general views (model windward side, side surfaces) and $7^{\circ}$-lens was used for observation of model fragments (flaps, nose bluntness, or base region).

The principal problem appeared in these tests was that IR camera frame rate was low and during tests (useful test duration was lower than $150 \mathrm{~ms}$ for all the flow regimes), only a few IR-images (4-5 per a test) were recorded. Hence, straightforward approach to specification of heat fluxes by solution of heat conduction equation (with Dirichlet condition, see, e.g., [5]) is inadmissible in this case. For this reason, heat flux to the surface was determined by the following procedure. Temporal variation of incident heat flux for solution of heat conduction equation was supposed to be self-similar and was derived from the calorimetric data; required normalization constant was obtained from temperature IR measurements by least-square method. This approach is quite adequate for pure laminar flows but for turbulent and transient flows, some additional corrections are required.

\subsection{Sensor Measurements}

Common-wide copper calorimetric sensors (copper cylindrical sleeve with a thermocouple fixed at its rear surface) were utilized at nose part of the model for measurements of heat fluxes at prescribed locations (see Fig. 5). Thermoresistive sensors (thin-film resistive thermometers) were used in the base region where heating level was expected to be relatively low.

\subsection{Measurement Uncertainties}

Uncertainties in temperature determination by IR measurements strongly depend on level of background reradiation from the walls of the test chamber and nozzle. This effect is determined by a number of factors: facility run-start conditions, flow regime parameters $T_{0}, P_{\text {pitot }}$, and model orientation. Estimation of maximum effect of the background radiation shows that it is (translating into temperature variation is $\delta T_{w}$ ) lower than $5 \mathrm{~K}$ for the windward side of the model and about $1 \mathrm{~K}$ for base region. Error in temperature specification during model heating has a minimum at initial model temperature and at $T_{w}>320 \mathrm{~K}$ may achieve value of $\delta T_{w} \sim 1-2 \mathrm{~K}$.

Another important issue affecting the uncertainties of obtained results is a limited resolution of the IR-system that can be subdivided onto temporal and geometric ones. Temporal resolution is connected to inertial operation of the receiving chain of the IR-system, spatial - to finite size of the model surface mapped to a single pixel of IR-image. For example, at distance of $1 \mathrm{~m}$ from 
IR-camera to the model, the spatial resolution of the system is 2.3 and $7 \mathrm{~mm}$ for 7 - and $20^{\circ}$-lenses, respectively. Therefore, the best resolution of the heat flux field to the model surface can be obtained making shots of its fragments by $7^{\circ}$-lens. Restricted resolution of the system contributes additional errors within the regions with peak-like distribution of heat fluxes and in zones where signal is distorted due to Lambert's law violation, i. e., near edges, etc.

Summing aforementioned and taking into account uncertainties in specification of model material thermophysical properties, it can be remarked that actual uncertainty in specification of heat flux in PGU-7 tests by IR-thermography is lower than $10 \%$ for windward side of the model and lower than $30 \%$ for back sides of the model for surface heating greater than $3-5 \mathrm{~K}$.

Analysis of accuracy of calorimetric measurements shows that relative error in specification of the heat flux $q_{w}$ is $\pm 15 \%$ and determined in general by measurements of electric signal subjected to influence of electric noise in acquisition chains. Limit of the thermoresistive sensor permitted inaccuracy is $10 \%$ at confidence probability of 0.95 .

\section{EXPERIMENTAL RESULTS}

For the sake of convenience, the obtained test data on heat fluxes were normalized to heat flux $\left(q_{w 0}\right)$ to the stagnation point of spheres with effective radius $R_{\text {eff }}$ equal to $70.5,69.9$, and $70 \mathrm{~mm}$ depending on attack angles of $40^{\circ}, 45^{\circ}$, and $50^{\circ}$, respectively. The value of $q_{w 0}$ was specified by well-known Fay and Riddel relationship [6]:

$$
q_{w 0}=0.763 \operatorname{Pr}^{-0.6}\left(\frac{\rho_{w} \mu_{w}}{\rho_{0} \mu_{0}}\right)^{0.1} \sqrt{\rho_{0} \mu_{0}\left(\frac{d u_{\delta}}{d s}\right)_{0}}\left(H_{\delta}-c_{p} T_{w}\right)
$$

for every test time moment $t$ by proper values of the inflow parameters. Convenience of such kind of data presentation is explained by conservative behavior of the relative value of $q_{w} / q_{w 0}$ for most part of the model surface at variation of the governing parameters $-\mathrm{M}_{\infty}, \operatorname{Re}_{L \infty}$, and $t_{w}=T_{w} / T_{0}$ (if the flow regime in the boundary layer remains the same).

\subsection{Windward Surface}

Infrared data on heat transfer for entire wwd surface were obtained using $20^{\circ}$ lens and close-up exposures (for zone of deflected flaps) were simultaneously made with the $7^{\circ}$-lens providing better spatial resolution of complex heat flux distributions in these zones. These data allow refining maximum values of $q_{w} / q_{w 0}$ within zones of increased heating at the flaps and locating position of these peaks. 


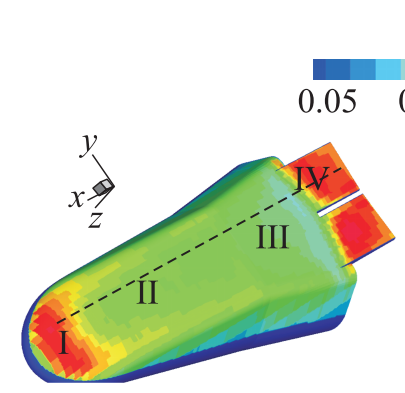

(a)

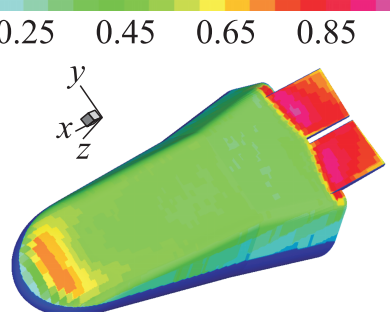

(b)

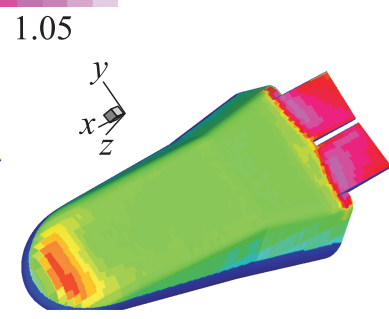

(c)

Figure 6 Windward distribution of normalized heat flux $\left(q_{w} / q_{w 0}\right)$ : (a) low Re mode; (b) medium Re mode; and (c) high Re mode $\left(\delta_{l}=\delta_{r}=15^{\circ}, \alpha=45^{\circ}, \beta=0^{\circ}\right)$. (Refer R. Kovalev et al., p. 344.)

Typical distribution of heat fluxes at the wwd side of the model is shown in Fig. 6 for three studied Re modes. There are several regions on the body with specific heating within: three-dimensional (3D) shaped nose bluntness (I, see Fig. 6), front flat surface (II) adjacent to the nose junked to the aft flat surface (III), and airbrake flap region (IV). Nose part heating in these general views is about $20 \%$ lower than in the case of close-up picture (see Fig. 5) because of insufficient spatial and dynamic resolution of this region with the $20^{\circ}$-lens and improper nose observation angle. Maximum heating on the flat surfaces is reached at the body shoulder edges where flow is accelerated towards the lateral surfaces of the model. This increase is about $10 \%-15 \%$ of average heating value in $x=$ const section. Also, there is a slight growth of heat fluxes near the junction line between front and aft flat surfaces induced by flow acceleration in Prandtl-Meyer rarefaction wave.

Local minimum of heat fluxes in front of the deflected flaps hinging line for low Re mode indicates that complex shaped laminar separation zone arises there. The size of this zone decreases rapidly when Reynolds number increases from low mode to medium and high modes. It also can be seen from Fig. 7 where longitudinal distributions of heat fluxes along off-center line (dashed line in Fig. $6 a$ ) extracted from $7^{\circ}$-lens close-up image are shown for three Re modes ( $x$-coordinate origin corresponds to the body base section). For medium and high Re modes, the separation zones appear to be transitional; moreover, for high Re mode, LTT on-set is located just behind two wwd flat surfaces junction line and transition starts before flow separation. For medium Re case, LTT seems to be provoked by the separation. With growth of the attack angle $\alpha$, the characteristic values of $q_{w} / q_{w 0}$ ratio for wwd surface also increases (approximately 1.2 times within the studied range of $\alpha=40^{\circ}-50^{\circ}$ ). Sideslip angle $\beta=5^{\circ}$ causes low asymmetry in $q_{w} / q_{w 0}$ on the windward side. In this case, heat fluxes at the 


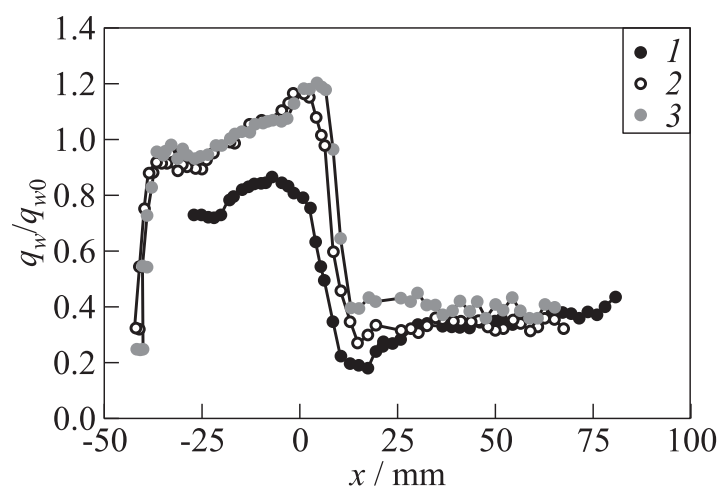

Figure 7 Heat flux distribution in near-flap zone $\left(\delta=15^{\circ}, \alpha=45^{\circ}\right): 1-$ low Re mode; 2 - medium Re mode; and 3 - high Re mode

body shoulder edges of the body side turned to the flow are $10 \%-15 \%$ greater than that of corresponding symmetric points of the leeward side. Also, heating of windward lateral side is $50 \%-80 \%$ greater than that of opposite leeward lateral surface.

\subsection{Flaps}

Heat flux distribution on the flap surface is quite nonuniform, especially in case of their differential deflection. Maximum values of heat fluxes for all Re modes are observed in the region of reattachment of separated boundary layer to the flap surfaces. Besides, there is another zone at internal flap edges near the gap between them where boundary layer flow is accelerated by strong pressure gradient inducing narrow peaks of intensive heat transfer. Measured values of $q_{w} / q_{w 0}$ over there exceed average level on the flap at $15 \%-20 \%$. The effect of flap deflection angle for low Reynolds number mode is demonstrated in Fig. 8 where normalized distribution of heat fluxes along a surface line parallel to the model symmetry plane and going through the center of a flap (dashed line in Fig. 6a) is presented for four tested flap deflection angles. These curves combine IR measurements obtained simultaneously with $20^{\circ}$-lens (wwd surface $x \geq 50 \mathrm{~mm}$ ) and $7^{\circ}$-lens (wwd surface $x \leq 50 \mathrm{~mm}$ and flaps). Such distribution of heat fluxes on the flap shows evidently that for lower deflections $\left(\delta=0^{\circ}, 5^{\circ}\right.$, and $\left.10^{\circ}\right)$, flow seems to remain laminar but in case of the highest deflection $\left(\delta=15^{\circ}\right)$, flow after reattachment becomes transitional.

It also can be revealed from consideration of characteristic values of heat fluxes to the flaps (Fig. 9) depending on deflection angle for all tests (at $\alpha$ $=45^{\circ}$ ). These characteristic values were obtained by averaging heat fluxes (to 


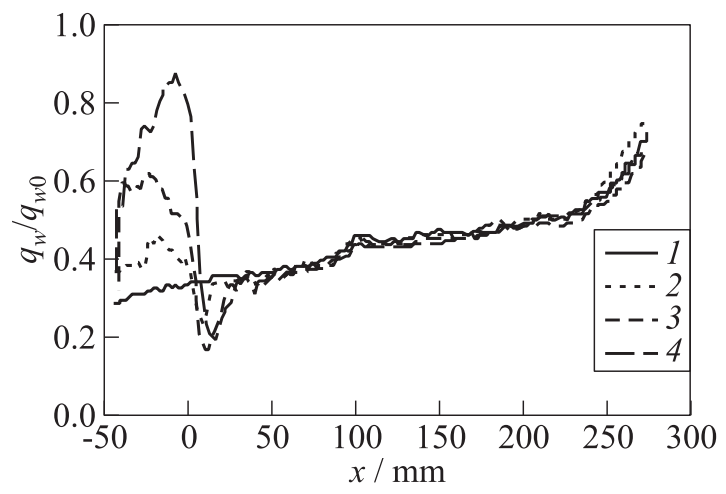

Figure 8 Off-centerline heat flux distribution (low Re mode, $\alpha=45^{\circ}$ ): $1-\delta=0^{\circ}$; $2-5^{\circ} ; 3-10^{\circ}$; and $4-\delta=15^{\circ}$

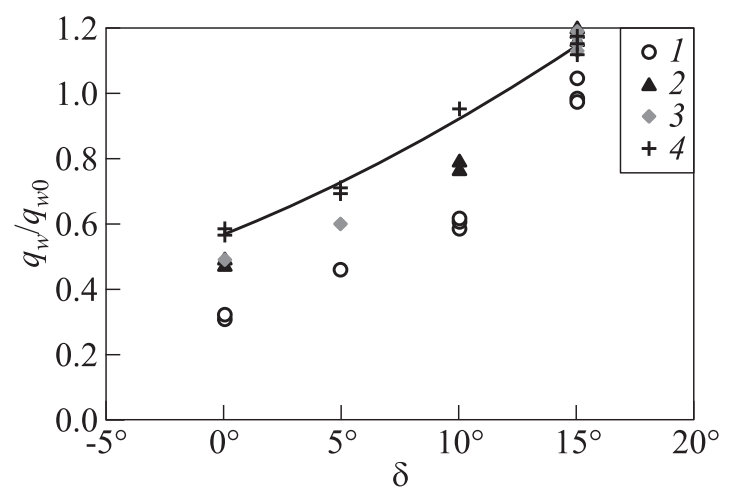

Figure 9 Characteristic values of heat flux at the flaps vs. deflection angle for different Re modes $\left(\alpha=45^{\circ}\right): 1$ - low Re mode; 2 - medium Re mode; 3 - high Re mode; and 4 - forced transition

reduce data scattering) over a small region near the flap center and approximately correspond to typical mean values of flap heating. The line in Fig. 9 is a trend line drawn through the data obtained for forced transition tests (4) and it corresponds to the turbulent heating magnitudes. Thus, only high Re mode provides fully turbulent heat transfer at the flaps in case of $\delta=15^{\circ}, \alpha=45^{\circ}$. For low $\left(\delta=15^{\circ}\right)$, medium, and high Re modes at $\delta<10^{\circ}$ cases, flow remains transitional also after reattachment. For lower angle of attack $\left(\alpha=40^{\circ}\right)$, flow even for $15^{\circ}$-deflected flaps appears to be pure laminar at low Re mode but fully turbulent for high Re mode. Maximum flap heating throughout the tests was observed in case of $\alpha=50^{\circ}, \delta=15^{\circ}$, and medium Re mode (for low Re mode, heating value 


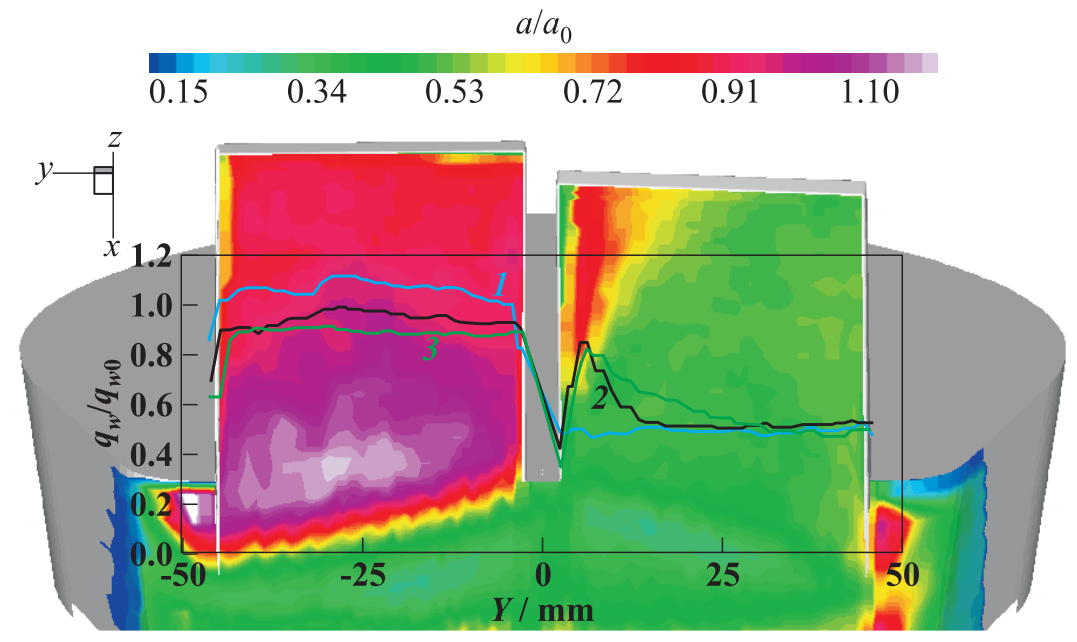

Figure 10 Heat flux to the differential flaps (medium Re mode, $\alpha=45^{\circ}, \delta_{r}=5^{\circ}$, and $\delta_{l}=15^{\circ}$ ): $1-x=11 \mathrm{~mm} ; 2-25.7$; and $3-x=40.3 \mathrm{~mm}$. (Refer R. Kovalev et al., p. 347.)

was only slightly lower); in this situation, heating level at the flaps was about $\sim 1.4 q_{w 0}$ and $20 \%$ greater than in corresponding high Re mode case.

In case of differential flaps (when they are used as ailerons for lateral maneuvers), the shock wave induced by more deflected flap interacts with the shock and boundary layer on less deflected one bringing about amplifying of heat fluxes in that zone up to the levels specific for more deflected flap (Fig. 10). In that case, IV type of shock wave-shock wave (SW-SW) interaction occurs at the less deflected flap producing supersonic jet impinging flap that generates narrow strip of elevated heating on the flap wall and it seems that this narrow region can be unresolved because of insufficient resolution of measuring tools. Also should be noted that for all cases of deflected flaps small spots of very intensive heating on the fuselage surface near the base section were registered in tests, heating intensity increases with the increase of deflection angle and may achieve $\sim(1.7-1.8) q_{w 0}$ for $\delta=15^{\circ}$. Obviously, intensification of heat transfer in that spots is explained by shock wave-boundary layer (SW-BL) interaction of shock wave induced by the deflected flap and thin boundary layer accelerated by low pressure in base region.

\subsection{Base Region}

Though measurements in this region were made for three considered Re modes, in most cases, the level of the IR-camera signal is too low and it cannot be 
often extracted from the background radiation level. It evidences on relatively low heating of the base region for the most of analyzed flow regimes. Only at highest Reynolds numbers, some increase of the heat fluxes in the base region were observed in zone where high-velocity streamline from the flap edges attaches the base section (Fig. 11).

The measurements of the thermoresistive sensors allow evaluating value of the heat fluxes in the base region. One of them was installed in the center of base (TB2 in Fig. 11) and another in the gap between flaps (TB1). For low Re mode, characteristic heating level is $\sim 0.1^{\circ}$ for TB2 sensor and $\sim 1^{\circ}$ for TB1 sensor and heat fluxes in this region are respectively 3 and 2 order lower than maximum heating level. For high Re mode, heat fluxes at these points are about $1 \%$ of $q_{w 0}(\mathrm{~TB} 1)$ and $10 \%-30 \%$ of $q_{w 0}$ (TB2). Also, another spot of

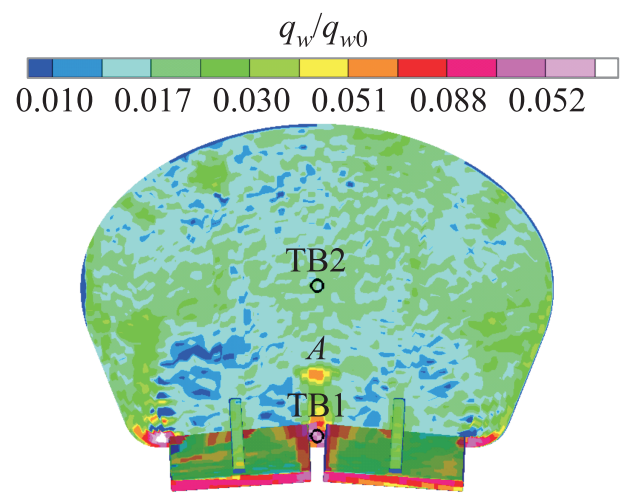

(a)

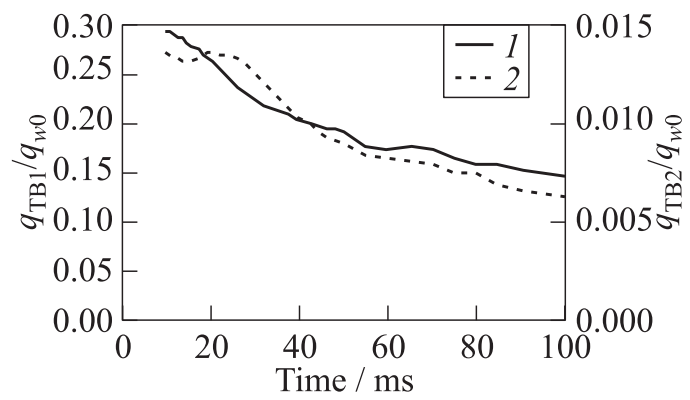

(b)

Figure 11 Heat flux distribution over base region of the model $(a)$ and sensor data $(b): 1-q_{\mathrm{TB} 1} / q_{w 0} ;$ and $2-q_{\mathrm{TB} 2} / q_{w 0}$. High Re mode, $\alpha=45^{\circ}, \delta=15^{\circ}$. (Refer R. Kovalev et al., p. 348.) 
relatively elevated heating ( $A$ in Fig. 11) was observed in the tests with heat flux $\sim(0.05-0.06) q_{w 0}$ for high Re mode. This spot corresponds to attachment of high-velocity stream line issued from the flap gap to the launcher interface frame structure (1 in Fig. $4 b$ ).

\section{CONCLUDING REMARKS}

A test campaign on studying ATD effects of the Pre-X configuration during reentry at Mach 10 has been carried out. Reynolds number sensitivity analysis with three different Reynolds numbers, several combinations of flap deflection angle, angle of attack, and sideslip configurations have been made. Heat flux determination by means of IR thermography and discrete sensors were the main measurement techniques. Results of conducted tests enable to reveal clearly heat transfer peculiarities on the PRE-X vehicle model, obtain heat flux distribution over its windward surface, locate most heated zones on deflected flaps, and estimate heat flux levels in them at different values of attack angles and sideslip.

Increase of Reynolds number leads to decreasing of separation zone in front of the flaps. Besides, separation (and, perhaps, following reattachment) is that zone where flow instabilities can be generated giving rise to LTT. At flap deflection $\delta=15^{\circ}$, separation induces transition for all three investigated Re modes that can be specified from sharp increase of heat fluxes just behind the flap hinging line. Intensive SW-SW and SW-BL interactions and acceleration of flow at the flaps and in their vicinity by lower pressure in the base region are the main reasons for arising narrow strips and relatively small spots of elevated heat fluxes on the model surfaces.

\section{ACKNOWLEDGMENTS}

The authors express their frank acknowledgement to L. Dedukov for his huge and efficient efforts on model drawing and preparation and to all the team of PGU-7 for their efficient work with respect to the facility operation. Also, this paper was prepared under sponsorship of ISTC foundation.

\section{REFERENCES}

1. Baiocco, P., S. Guedron, P. Plotard, and J. Moulin. 2006. The Pre-X atmospheric re-entry experimental lifting body: Program status and system synthesis. 57th Astronautical Congress (International). Valencia, Spain. 
2. Anfimov, N.A., and V.V. Kislykh. 1989. Multi-cascade compression - effective means to obtain high temperature dense gas in piston gas dynamic units (PGU). Current topics in shock waves: 17th Symposium (International) on Shock Waves and Shock Tubes. Ed. Y.W. Kim. Bethlehem, P.A.

3. Anfimov, N. A., V. V. Kislykh, and B. A. Zemliansky. 1999. Methods and means for studying flying vehicles heat transfer at hypersonic velocities. AIAA Paper No. 994891.

4. AGA Thermovision 780. Operating Manual. 1980. Publication No. 556556492. Ed. II. AGA Infrared Systems AB.

5. Neeb, D., A. Gülhan, E. Cosson, R. Ameziane, P. Binetti, and T. Walloschek. 20082009. An experimental study on aerothermal heating of the IXV configuration during re-entry. 6th European Symposium on Aerothermodynamics for Space Vehicles. Versailles, France.

6. Fay, J. A., and F. R. Riddel. 1958. Theory of stagnation point heat transfer in dissociated air. J. Aeronaut. Sci. 25(2):73-85, 121. 